\title{
Is ultrasound elastography adding value in diagnosis of focal hepatic lesions? Our experience in a single-center study
}

\author{
Doaa M. Emara ${ }^{1,2^{*}}$, Mohamed M. El Shafei ${ }^{1}$, Ahmed El-Gendi ${ }^{3}$ and Amany A. Yousif ${ }^{4}$
}

\begin{abstract}
Background: The aim of this study was to assess the use of ultrasound elastography in differentiating hepatic lesions in order to increase the sensitivity and specificity of grey-scale ultrasound.

Methods: This study included 104 patients who were referred to the radiology department at Alexandria Main University Hospital for focal hepatic lesions detected by grey-scale ultrasound and were recommended to undergo further evaluation by ultrasound elastography. All studied patients were subjected to the following: (1) grey-scale ultrasound and ultrasound elastography using semiquantitative technique and (2) triphasic MDCT of the liver. MRI was done in 11 patients with malignant lesions; further confirmation with histopathological assessment was conducted.

Results: Benign lesions showed a low strain ratio, while malignant lesions showed a high strain ratio. The mean ratio in the benign lesions was $1.08 \pm 0.40$, while the mean ratio in the malignant lesions was $4.14 \pm 1.25$. The cut-off value used to diagnose the malignant lesions and differentiate these lesions from the benign lesions was 1.7, which had a sensitivity of $100 \%$, specificity of $93.10 \%$, PPV of $97.40 \%$ and NPV of $100 \%$.
\end{abstract}

Conclusion: Ultrasound elastography is a promising non-invasive, non-contrast technique that can be added to routine grey-scale sonographic examinations of the liver to characterize hepatic lesions.

Keywords: Ultrasound, Elastography, Focal hepatic lesions, Stiffness, Strain ratio

\section{Background}

In our practice, focal hepatic lesions constitute a daily challenge; thus, our aim is to use the least invasive imaging techniques to characterize and diagnose these lesions [1].

A differential diagnosis of focal hepatic lesions using different imaging modalities depends on the surrounding liver parenchyma, mainly the presence of cirrhosis, the clinical data of the patient and the diagnostic imaging findings of the mass [2].

Currently, different imaging techniques are used to examine the liver, and ultrasonography is the main modality used to detect additional hepatic lesions [3].

\footnotetext{
* Correspondence: dr.emara-doaa@hotmail.com; dr.emara_doaa@yahoo.com 'Department of Radiodiagnosis, Faculty of Medicine, Alexandria University, Alexandria, Egypt

${ }^{2}$ Department of Diagnostic and Interventional Radiology, Faculty of Medicine, Alexandria University Hospital, Champollion Street, El Azareeta, Alexandria, Egypt

Full list of author information is available at the end of the article
}

Ultrasound is typically used as the basic imaging study to detect focal hepatic lesions because it is a widely available, non-invasive, safe, low-cost technique. However, the main disadvantage of ultrasound is the inability to characterize and differentiate hepatic masses. Thus, unfortunately, ultrasound has a low sensitivity and specificity of less than $70 \%$ in characterizing hepatic lesions $[4,5]$.

Recently, non-invasive novel stiffness-based imaging techniques, such as MRI and ultrasound elastography, have been introduced to assess the degree of stiffness in hepatic lesions; these techniques can be used in the liver and other organs, such as the breast and thyroid. Different ultrasound elastography techniques exist, including shear wave elastography and acoustic radiation force impulse (ARFI) elastography [6].

The most commonly used ultrasound elastography techniques include strain elastography (SE) and shear wave elastography (SWE). Both techniques assess the degree of stiffness but use different methods [7]. 
Table 1 Distribution of the studied cases according to demographic data $(n=104)$

\begin{tabular}{ll}
\hline & No. (\%) \\
\hline Mex & \\
Male & $45(43.3 \%)$ \\
Age (years) & $59(56.7 \%)$ \\
$\leq 50$ & $47.55 \pm 14.71$ \\
$>50$ & $49(47.1 \%)$ \\
\hline
\end{tabular}

The use of ultrasound elastography was approved by the Food and Drug Administration. Currently, ultrasound elastography is a popular technique that is widely used in many clinical applications, including in the differentiation of hepatic lesions [7].

Ultrasound elastography is a non-invasive, noncontrast, rapid easily applicable technique that is easy to perform as a continuation of routine ultrasound examinations of the liver. Ultrasound elastography increases the sensitivity and specificity of ultrasound in the differentiation of focal hepatic masses $[8,9]$.

Using ultrasound elastography, the lesion stiffness can be qualitatively expressed via a colour-coded map or semi-quantitatively via the strain ratio [5].

One of the ultrasound elastography techniques expresses the stiffness of the lesion in the form of a strain ratio, which is the ratio of the tension (stress) needed to produce a relative change in length (strain). Different
Table 2 Comparison between benign and malignant lesions according to the strain ratio

\begin{tabular}{llll}
\hline Strain ratio & Benign $(n=29)$ & Malignant $(n=75)$ & $p$ \\
\hline Min.-Max. & $0.60-2.0$ & $1.80-7.0$ & $<0.001^{*}$ \\
Mean \pm SD & $1.08 \pm 0.40$ & $4.14 \pm 1.25$ & \\
Median & 1.0 & 3.90 & \\
\hline $\begin{array}{l}\text { * value for Mann-Whitney test } \\
\text { *Statistically significant at } p \leq 0.05\end{array}$ & & \\
& & &
\end{tabular}

agents can affect tissue elasticity, including ageing, inflammation and malignancies. Malignant lesions are commonly hard, less compressible and are less strained than softer tissues under the same pressure [5].

\section{Aim of the study}

The aim of this study was to assess the ability of ultrasound elastography, which is a type of stiffness-based imaging, to differentiate focal hepatic lesions to increase the sensitivity and specificity of grey-scale ultrasound.

\section{Methods}

This prospective study included 104 patients, who were referred to the radiology department at Alexandria Main University Hospital for focal hepatic lesions detected by grey-scale ultrasound and were recommended to undergo further evaluation by ultrasound elastography. This study was performed between December 2015 and June 2016.

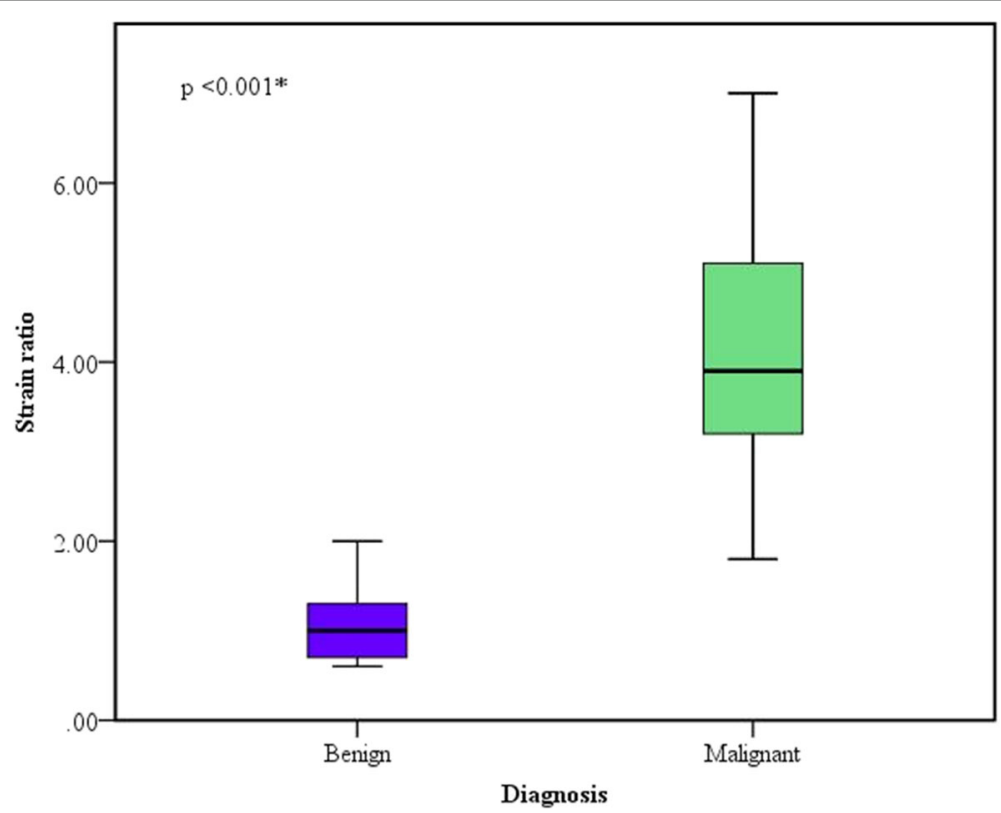

Fig. 1 Comparison of benign and malignant lesions according to the strain ratio 
The study protocol was approved by the Research Review Committee of the Alexandria Faculty of Medicine, and informed consent was obtained from each subject included in the study.

All studied patients were subjected to the following:

1. Grey-scale ultrasound;

2. Ultrasound elastography using a semiquantitative technique to compare the stiffness of the lesion to that of the surrounding liver parenchyma. A green signal on the right side of the image was an indicator of adequate compression;

3. Triphasic MDCT of the liver to further characterize the hepatic lesions. In 11 patients ( 9 patients with focal nodular hyperplasia (FNH) and 2 patients with adenoma), further assessment using triphasic MRI was conducted; and

4. In malignant lesions, histopathological assessment was conducted for further confirmation.

\section{Statistical analysis}

The data was collected and entered into the personal computer. Statistical analysis was done using Statistical Package for Social Sciences (SPSS/version 20) software.

Arthematic mean, standard deviation and MannWhitney test were used.

\section{Results}

This study included 104 patients, including 45 males and 59 females with a mean age of $47.55 \pm 14.71$ years (Table 1).

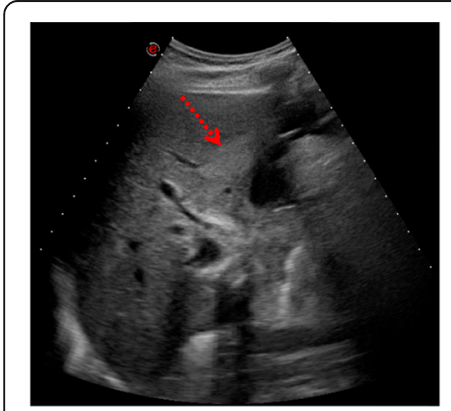

$\mathbf{a}$

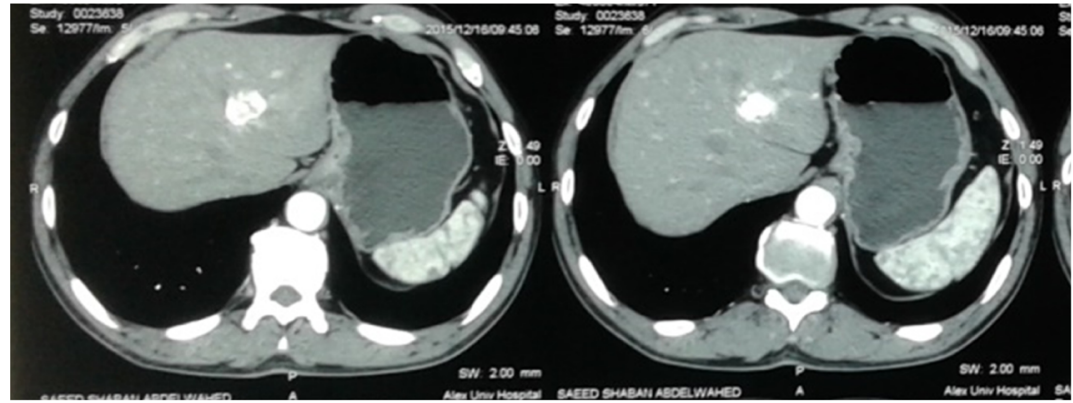

d

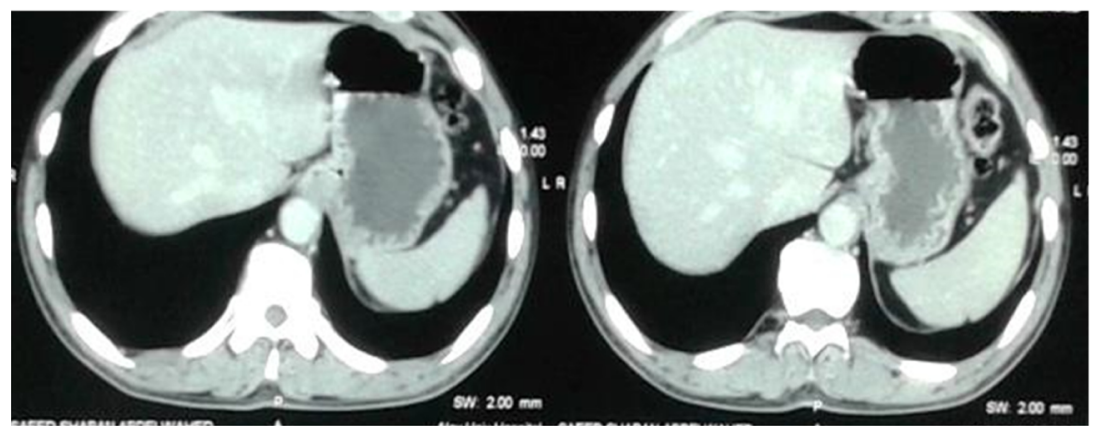

e

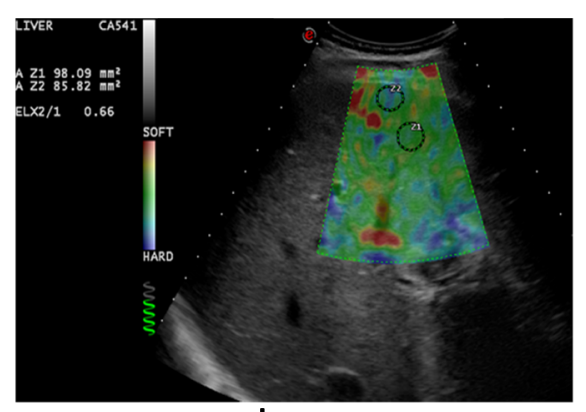

b

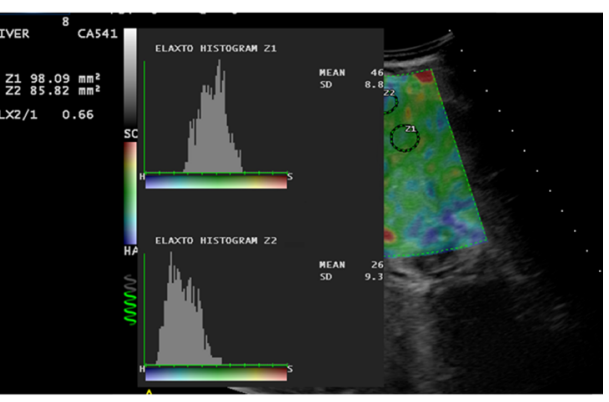

C

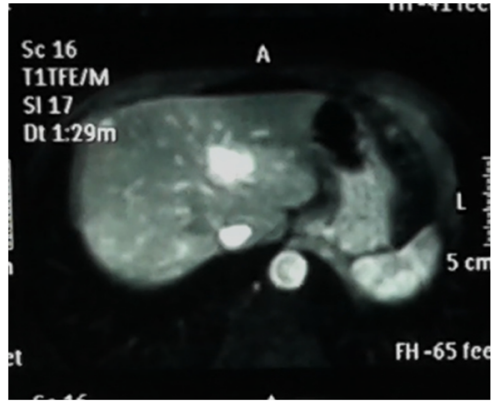

f

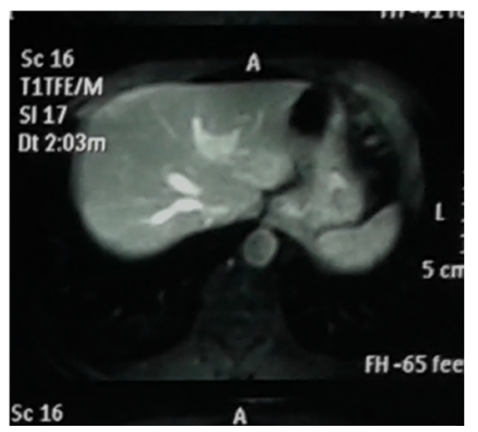

g

Fig. 2 A 38-year-old female patient with a well-defined hyperechoic lesion as determined by US. Arrow shown in a indicates a strain ratio of 0.66 by elastography (b) and a histogram of the lesion (c), HAP CT and MRI (d, f), PVP CT and MRI (e, g) showing homogenous enhancement of the lesion. This lesion was diagnosed as hepatic haemangioma 


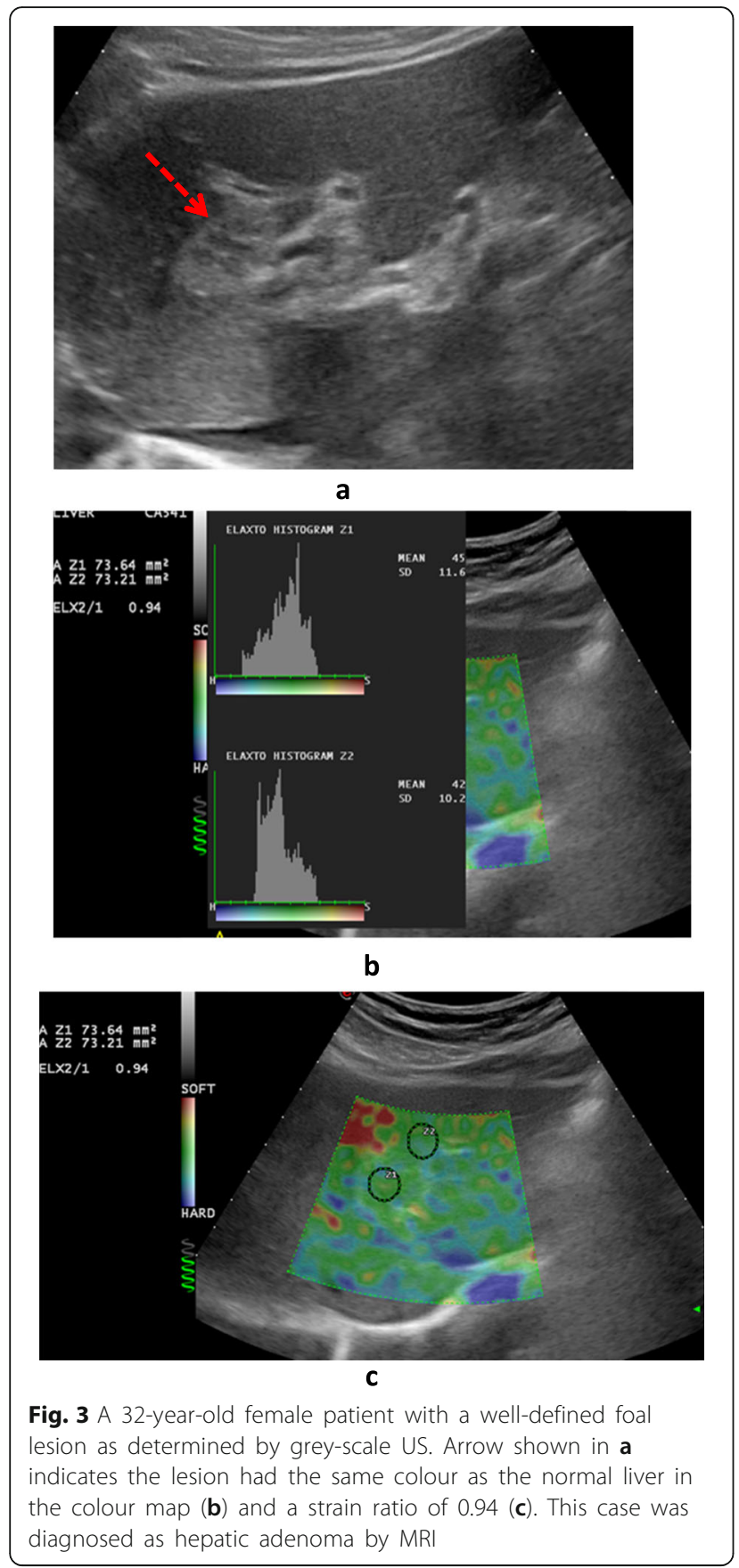

\section{Strain ratio in the studied lesions}

The benign lesions showed a low strain ratio, while the malignant lesions showed a high strain ratio. The mean ratio in the benign lesions was $1.08 \pm 0.40$, while the mean ratio in the malignant lesions was $4.14 \pm 1.25$, and the difference in the strain ratios between the benign and malignant lesions was statistically significant $(p \leq 0.05)$ (Table 2 and Fig. 1).

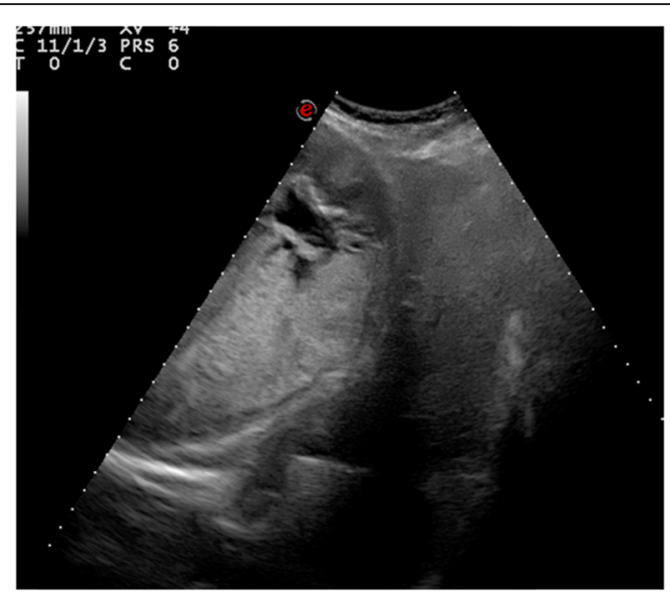

a

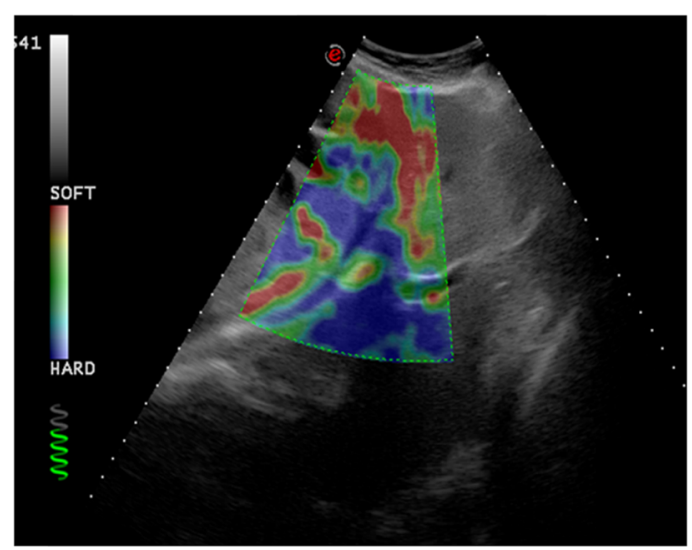

b

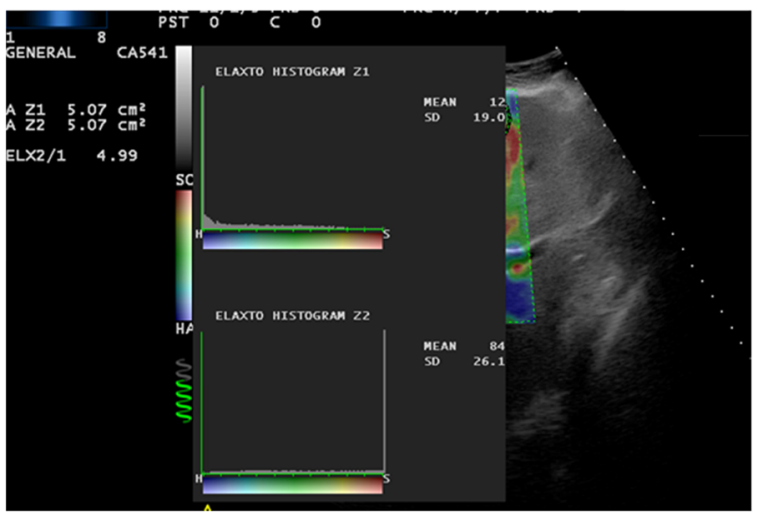

C

Fig. 4 A 48-year-old male patient with a well-defined FHL as determined by ultrasound (a) with a strain ratio of 4.9 as shown by ultrasound elastography (b), the histogram of the lesion (c) and the pathological finding of cholangiocarcinoma

\section{Strain ratio in the benign lesions}

In the benign lesions (29 cases), the lowest ratio was found in cases with haemangiomas (18 cases), which had a mean ratio of $0.82 \pm 0.20$ (Fig. 2), followed by 


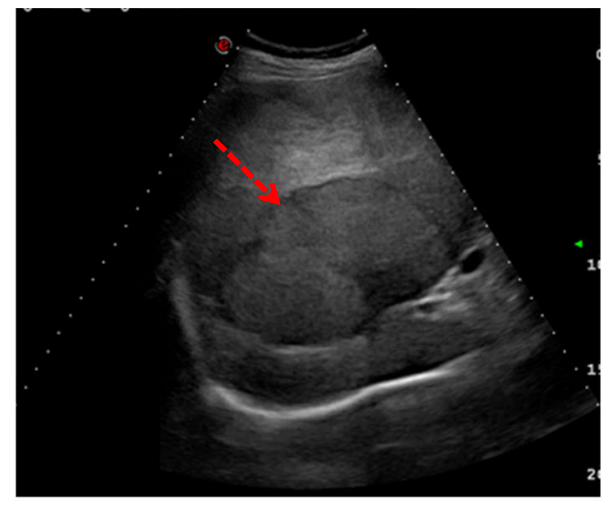

a

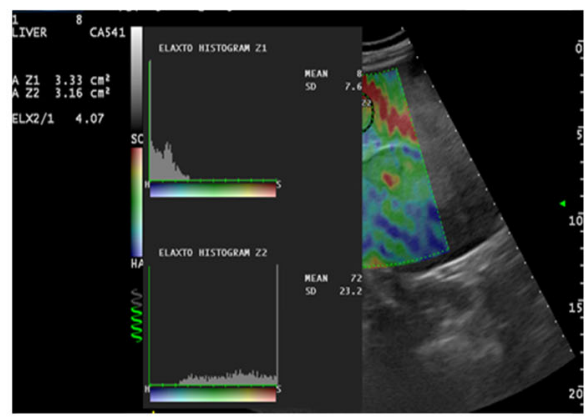

b

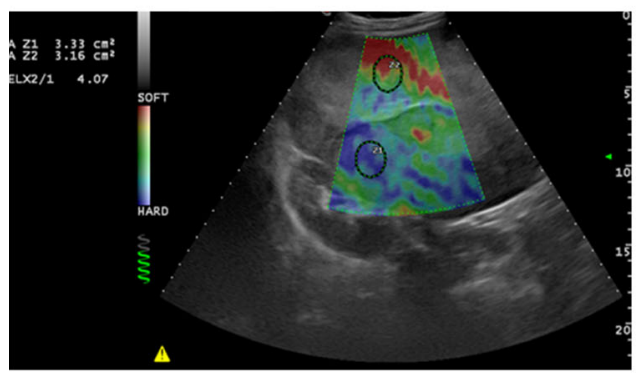

C

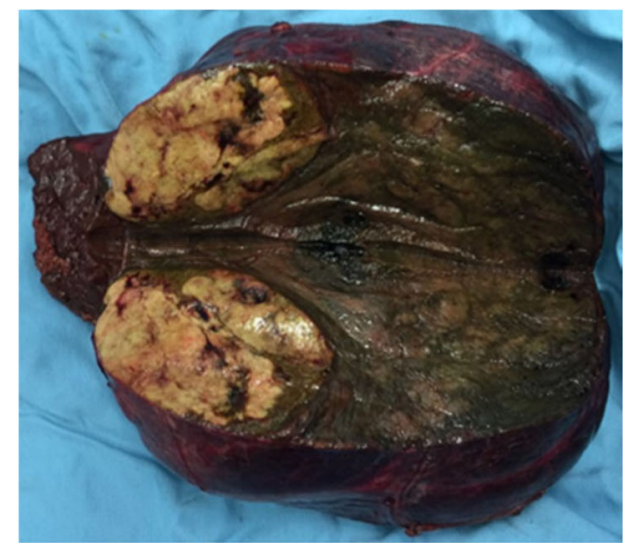

d
Fig. 5 A 12-year-old female patient with a rather well-defined focal hepatic lesion as determined by grey-scale US that was (a) variable in stiffness but mainly hard with a strain ratio of 4.07 (b), histogram of the mass lesion (c). This lesion was surgically removed and diagnosed as hepatoblastoma (d)

adenomas ( 2 cases), which had a mean ratio of $1.30 \pm$ 0.14 (Fig. 3) and, finally, FNH (9 cases), which had a mean ratio of $1.54 \pm 0.27$.

\section{Strain ratio in the malignant lesions}

In the malignant lesions (75 cases), the highest strain ratio was found in cases with cholangiocarcinoma (10 cases), which had a mean ratio of $6.25 \pm 0.44$ (Fig. 4), followed by hepatoblastoma (4 cases), which had a mean ratio of $4.05 \pm 0.29$ (Fig. 5), hepatocellular carcinoma (HCC) (40 cases), which had a mean ratio of $3.85 \pm 1.14$ (Fig. 6) and, finally, metastasis (21 cases), which had a mean ratio of $3.72 \pm 0.72$ (Fig. 7 ).

The mean strain ratios in each lesion are summarized in Table 3 and Fig. 8.

The cut-off value used to diagnose the malignant lesions and differentiate these lesions from the benign lesions was 1.7 , which had a sensitivity of $100 \%$, specificity of $93.10 \%$, PPV of $97.40 \%$ and NPV of $100 \%$ (Table 4 and Fig. 9). The management of our cases was performed as follows: patients with benign lesions, including adenomas, FNH and haemangiomas, were scheduled to undergo non-surgical regimens with routine followup. In contrast, in the malignant cases, the management was tailored to each case. For patients with HCC, 25 cases were managed by TACE, 10 cases were managed with surgical resection and 5 cases with malignant portal vein thrombosis were candidates for chemotherapy. All cases with hepatoblastoma were managed surgically.

The 21 cases with hepatic metastases were managed as follows: 4 cases with solitary lesions were managed with surgical resection, while the other 17 cases with multiple lesions were scheduled to undergo chemotherapy.

\section{Discussion}

As radiologists, our aim is to diagnose the hepatic lesion and differentiate the benign masses from malignant masses using a non-invasive imaging technique with a high accuracy to avoid invasive procedures, such as liver biopsies [10].

Although ultrasound elastography has been used in different studies to assess the degree of hepatic fibrosis, few studies have used ultrasound elastography to differentiate focal hepatic masses according to their stiffness [4]. 


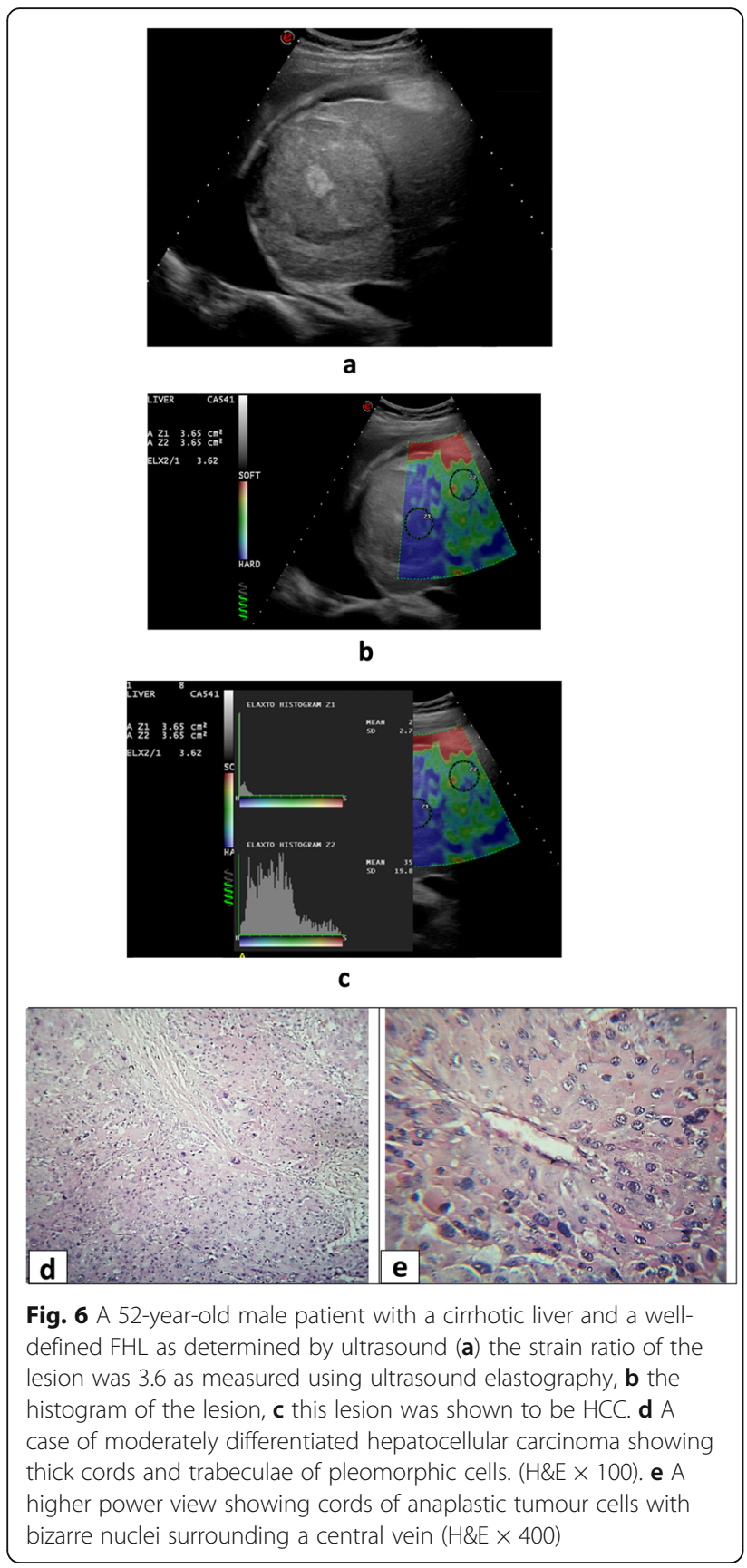

Different ultrasound elastography techniques are available; however, in the current study, we used a semiquantitative method using a strain index to express the elasticity of the lesion in relation to the surrounding normal liver parenchyma. This method is consistent with the semi-quantitative method used by Mehmet et al. [5] in 2013. In contrast, Hana et al. [2]. used ARFI to differentiate hepatic masses. Aymeric et al. used shear wave elastography [4].

In our study, the highest strain index was detected in cholangiocarcinoma (mean strain index $=6.25 \pm 0.44$ ),

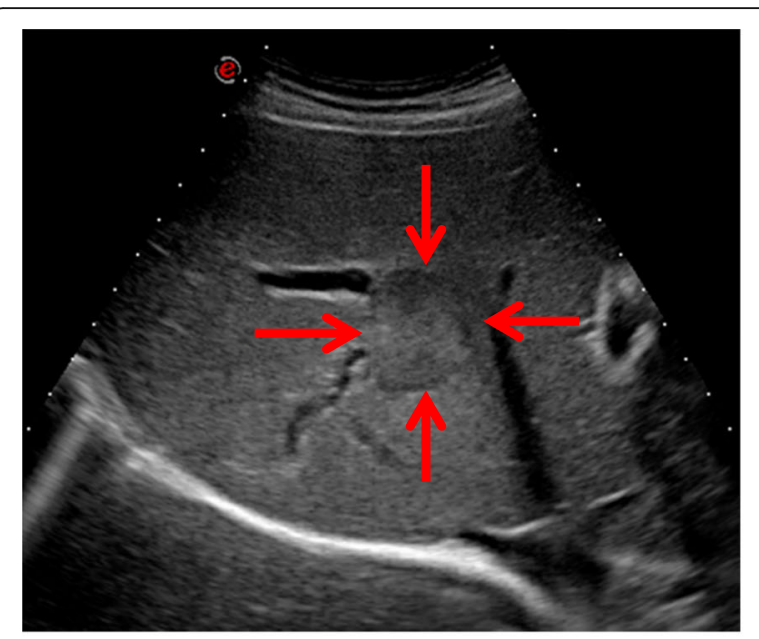

a

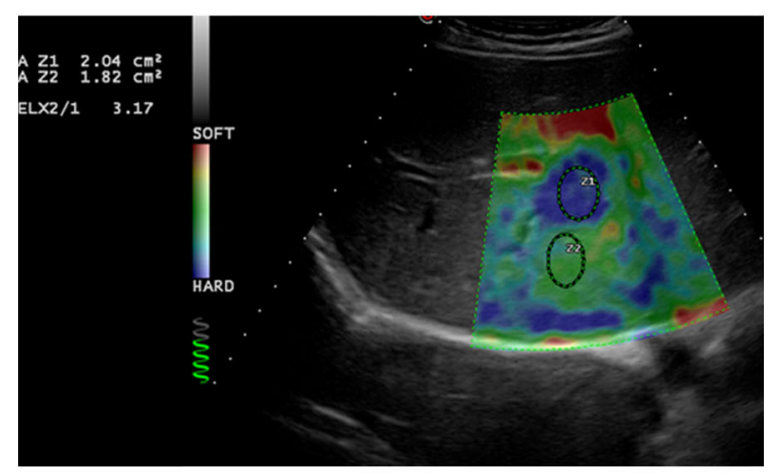

b

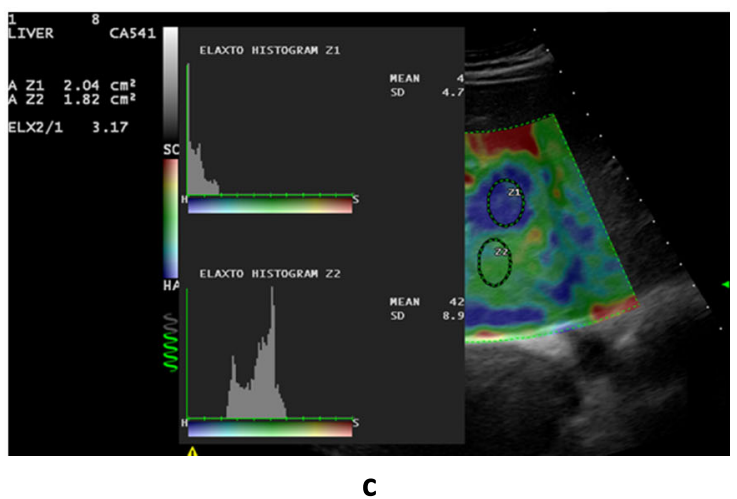

Fig. 7 A male patient with colon cancer and a solitary hepatic lesion as detected by US. Arrows shown in a indicate the stiffness by elastography, blue in the colour map (b) with a strain ratio of 3.17 (c). The pathology confirmed the lesion to be metastatic

followed by hepatoblastoma, HCC and hepatic metastases. Metastatic lesions from colon cancer had a higher strain index than other metastases or cystic metastases due to fibrosis, which increased the stiffness of the lesion. Using semi-quantitative strain ratio or shear wave 
Table 3 Relation between diagnoses with strain ratio

\begin{tabular}{lll}
\hline Diagnosis & $N$ & Strain ratio \\
\hline Cholangiocarcinoma & 10 & $6.25 \pm 0.44$ \\
Hepatoblastoma $^{\mathrm{a}}$ & 4 & $4.05 \pm 0.29$ \\
HCC $^{\mathrm{a}}$ & 40 & $3.85 \pm 1.14$ \\
Mets $^{\mathrm{a}}$ & 21 & $3.72 \pm 0.72$ \\
Hemangioma $^{\mathrm{ab}}$ & 18 & $0.82 \pm 0.20$ \\
FNH $^{\text {ab }}$ & 9 & $1.54 \pm 0.27$ \\
Adenoma $^{\text {ab }}$ & 2 & $1.30 \pm 0.14$ \\
\hline
\end{tabular}

elastography, Aymeric et al. [4], Mehmet et al. [5] and Ji et al. [6] concluded that cholangiocarcinoma and colon cancer metastases had the highest stiffness.

The strain index of the cholangiocarcinoma lesions in our study was $6.25 \pm 0.44$, which was the highest value among the studied cases. Consistent with Mehmet et al. [5], we found the highest strain index in malignant lesions, particularly in cases with cholangiocarcinoma.

Aymeric et al. [4], Mehmet et al. [5] and Ji et al. [6] detected a higher degree of stiffness in hepatic metastases from colon cancer than that in metastases from other primary tumours due to the presence of fibrous tissue. In addition, we found a high strain index in hepatic metastases from colon cancer reaching up to 5 , and this index was third highest in stiffness following hepatoblastoma and HCC.
Table 4 Agreement (sensitivity, specificity) for the strain ratio to diagnose malignant lesions

\begin{tabular}{llllll}
\hline & Cutoff & Sensitivity & Specificity & PPV & NPV \\
\hline Strain ratio & $>1.7$ & 100.0 & 93.10 & 97.40 & 100.0 \\
\hline
\end{tabular}

We found a high strain index in HCC, which is a malignant lesion that is hard in consistency, reaching a value of $3.85 \pm 1.14$. Mehmet et al. [5] confirmed the stiffness of $\mathrm{HCC}$ with a high strain ratio of $3.24 \pm 0.48$.

Benign lesions, such as hepatic haemangiomas, adenomas and $\mathrm{FNH}$, had a lesser degree of stiffness with a mean strain index of $0.82 \pm 0.20,1.30 \pm 0.14$ and $1.54 \pm 0.27$, respectively. This finding was consistent with reports by Mehmet et al. [5] who concluded that these benign lesions had a low strain index of $0.92 \pm 0.14$ in haemangioma and $1.75 \pm 0.16$ in $\mathrm{FNH}$.

Mehmet et al. [5] found that the sensitivity and specificity of conventional ultrasound in differentiating benign and malignant hepatic lesions ranged from 28.1 to $58.8 \%$ and 34.6 to $50.7 \%$, respectively. In their study, the authors attained a higher sensitivity and specificity using semi-quantitative strain elastography than those attained using conventional sonography. In addition, we attained a higher sensitivity and specificity reaching 100\% and $93 \%$, respectively, in differentiating benign from malignant hepatic lesions using strain elastography.

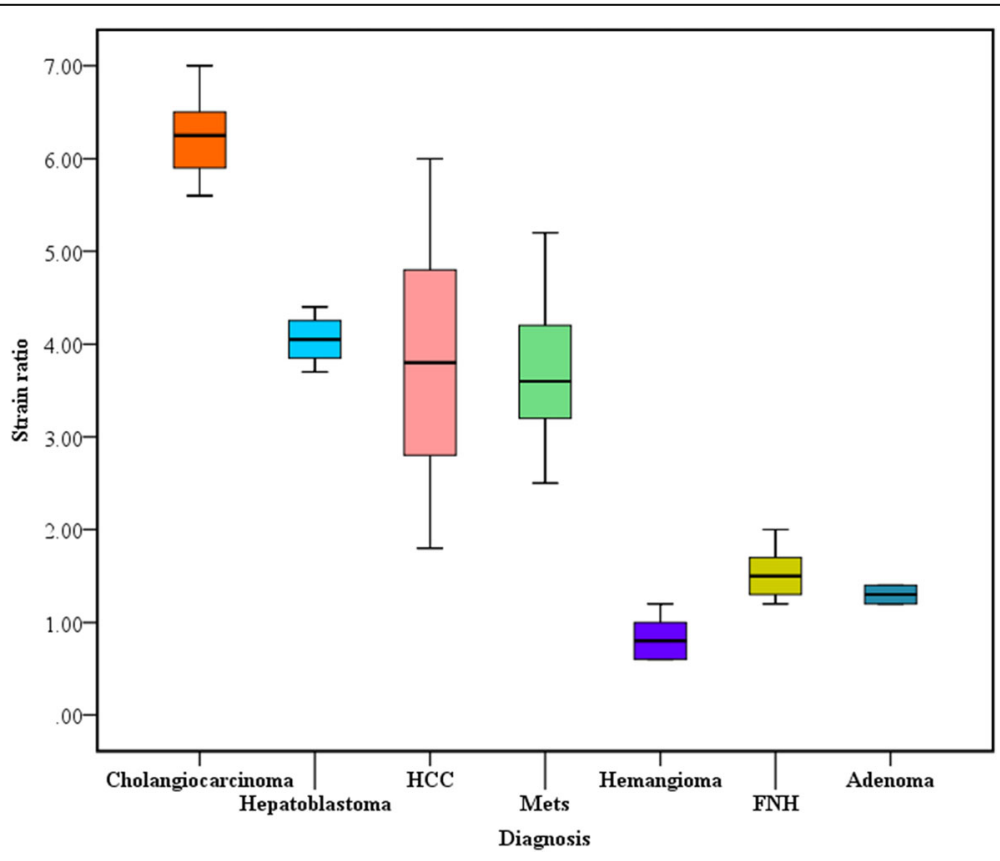

Fig. 8 Relationship between diagnoses and strain ratios 


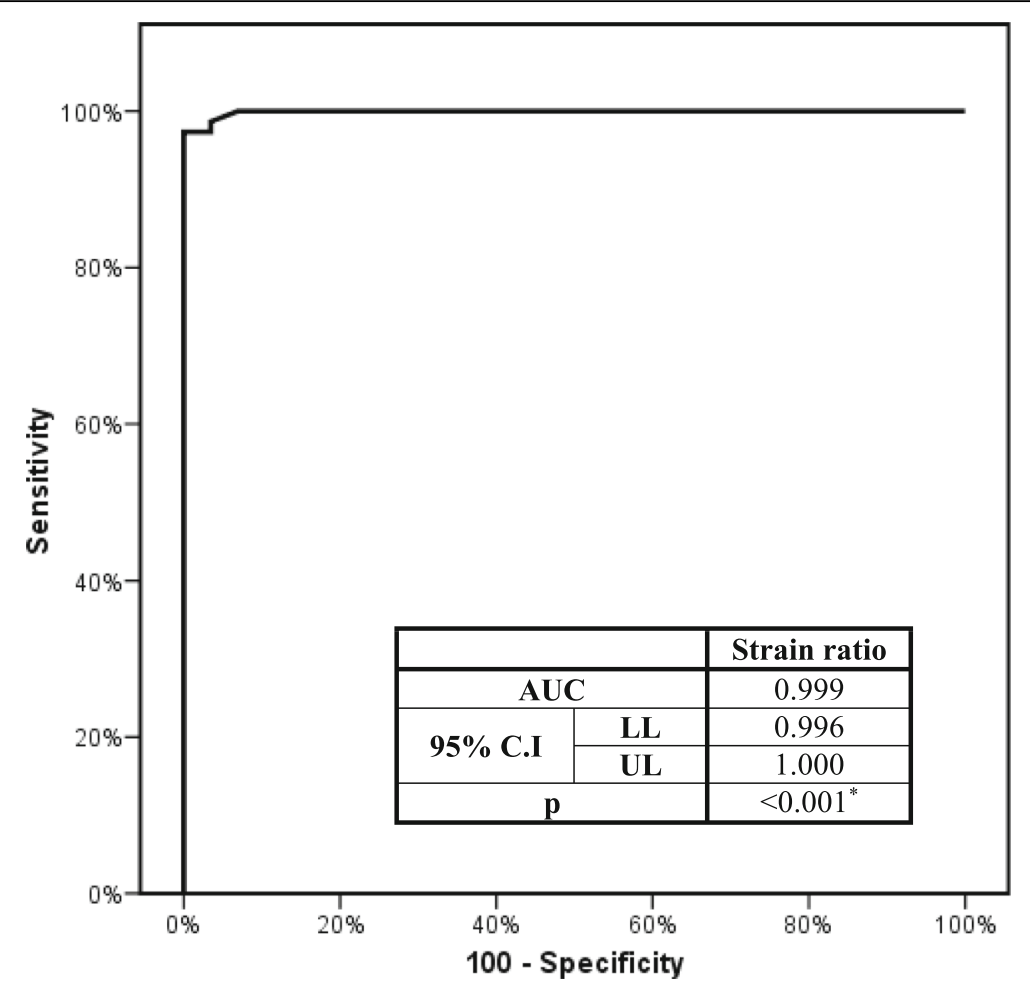

Fig. 9 ROC curve of the strain ratio used to diagnose malignant lesions

\section{Conclusions}

Ultrasound elastography is a promising, non-invasive, non-contrast technique that can be added to routine grey-scale sonographic examinations of the liver to characterize hepatic lesions. However, the heterogeneity of the lesions, i.e. malignant lesions with necrosis or benign lesions with fibrosis, should be considered because these characteristics can change the stiffness of the lesion as measured by elastography and can lead to false positive or false negative results.

\section{Abbreviations}

ARFI: Acoustic radiation force impulse; FNH: Focal nodular hyperplasia; HCC: Hepatocellular carcinoma; MDCT: Multidetector computed tomography; MRI: Magnetic resonance imaging; SE: Strain elastography; SWE: Shear wave elastography

\section{Acknowledgements}

"Not applicable"

\section{Authors' contributions}

$\mathrm{DE}$ and $\mathrm{ME}$ drafted the manuscript and edited the images. AE reviewed the clinical part of the work. AY revised the pathology of the cases. All authors read and approved the final manuscript.

\section{Funding}

"Not applicable"

\section{Availability of data and materials}

The datasets used and/or analysed during the current study are available from the corresponding author on reasonable request.
Ethics approval and consent to participate

Approval for this cross-sectional prospective study was obtained from the Research Ethics Committee of Alexandria Faculty of Medicine (Ethics committee's reference number: 0302521, IRB No: 00007555- FWA No: 00015712). All study procedures were carried out in accordance with the Declaration of Helsinki regarding research involving human subjects. Written informed consent was obtained from the patients.

\section{Consent for publication}

Written informed consent for publication of this data was given by the patients.

\section{Competing interests}

The authors declare that they have no competing interests.

\section{Author details}

'Department of Radiodiagnosis, Faculty of Medicine, Alexandria University, Alexandria, Egypt. ${ }^{2}$ Department of Diagnostic and Interventional Radiology, Faculty of Medicine, Alexandria University Hospital, Champollion Street, El Azareeta, Alexandria, Egypt. ${ }^{3}$ Department of General Surgery, Faculty of Medicine, Alexandria University, Alexandria, Egypt. ${ }^{4}$ Department of Pathology, Faculty of Medicine, Alexandria University, Alexandria, Egypt.

Received: 8 December 2019 Accepted: 10 December 2019 Published online: 26 December 2019

\section{References}

1. Elsayes KM, Narra VR, Yin Y, Mukundan G, Lammle M, Brown JJ (2005) Focal hepatic lesions: diagnostic value of enhancement pattern approach with contrast-enhanced 3D gradient-echo MR imaging. Radiographics 25:12991320

2. Hana P, Jun Y, Do Y, Sang H, Chae Y, Kwang H, Seung U (2013) Characterization of focal liver masses using acoustic radiation force impulse elastography. World J Gastroenterol 19(2):219-226

3. Yousef M, Elsharkawy A, El Beshlawy M, Esmat G, Salama Z (2013) Use of ultrasonic transient elastography (Fibroscan) in the assessment of hepatic focal lesion stiffness. Open J Gastroenterol 3:107-112 
4. Aymeric $G$, Camille B, Matthew B, Mélanie V, Frank P, Thomas W, Jean $Y$, Nawele B, Jérôme D, Thibaut L (2013) Evaluation of shearwave elastography for the characterization of focal liver lesions on ultrasound. Eur Radiol 23: 1138-1149

5. Mehmet R, Ahmet K, Esra E, Zulkif B, Ibrahim H, Erkin O (2012) Semiquantitative strain elastography of liver masses. J Ultrasound Med 31: 1061-1067

6. Ji E, Jae Y, Kyung S, Joon K, Byung I (2013) Acoustic radiation force impulse elastography for focal hepatic tumors: usefulness for differentiating hemangiomas from malignant tumors. Korean J Radiol 14(5):743-753

7. Barr RG (2014) Elastography in Clinical Practice. Radiol Clin N Am 52(6): $1145-1162$

8. Inoue Y, Takahashi M, Arita J et al (2010) Intra-operative freehand real-time elastography for small focal liver lesions: "visual palpation" for non-palpable tumors. Surgery 148:1000-1011

9. Havre RF, Elde E, Gilja OH et al (2008) Freehand real-time elastography: impact of scanning parameters on image quality and in vitro intra- and interobserver validations. Ultrasound Med Biol 34:1638-1650

10. Qiang L, Wenwu L, Changli L, Jiawu L, Lin M, Jierong Q et al (2015) Hepatocellular carcinoma: stiffness value and ratio to discriminate malignant from benign focal liver lesions. Radiology 275:880-888

\section{Publisher's Note}

Springer Nature remains neutral with regard to jurisdictional claims in published maps and institutional affiliations.

\section{Submit your manuscript to a SpringerOpen ${ }^{\circ}$ journal and benefit from:}

- Convenient online submission

- Rigorous peer review

- Open access: articles freely available online

- High visibility within the field

- Retaining the copyright to your article

Submit your next manuscript at $\boldsymbol{\nabla}$ springeropen.com 